\title{
Successful management of infected tibia IL nail by debridement, lavage, and antibiotic impregnated v-nail
}

\author{
Satish R. Gawali ${ }^{*}$, Vinod Barve ${ }^{2}$, Shashikant B. Kukale ${ }^{3}$, Pramod V. Niravane ${ }^{4}$ \\ ${ }^{1}$ Assistant Professor, Department of Orthopedics, Government Medical College and Hospital, Latur, Maharashtra, \\ India \\ ${ }^{2}$ Senior Resident, Department of Orthopedics, Government Medical College and Hospital, Latur, Maharashtra, India \\ ${ }^{3}$ Assistant Professor, Department of Orthopedics, Government Medical College and Hospital, Latur, Maharashtra, \\ India \\ ${ }^{4}$ Junior Resident III, Department of Orthopedics, Government Medical College and Hospital, Latur, Maharashtra, India
}

Received: 25 June 2016

Accepted: 29 June 2016

*Correspondence:

Dr. Satish. R. Gawali,

E-mail: satishgawali61@gmail.com

Copyright: () the author(s), publisher and licensee Medip Academy. This is an open-access article distributed under the terms of the Creative Commons Attribution Non-Commercial License, which permits unrestricted non-commercial use, distribution, and reproduction in any medium, provided the original work is properly cited.

\section{ABSTRACT}

Background: Tibia is a most common subcutaneous long bone which is highly vulnerable for injury. Infected ILN tibia and non-union of tibia is much common in clinical practice which calls for challenging management protocols to control intramedullary infection. Considerable judgment is required to treat an infection in fracture with implant in situ. Mechanical stability and control of infections are two main factors in management of infected tibia with implant in situ. To remove infected material and bio-film by serial reaming of medullary canal, thorough lavage and local debridement of infected fracture site and sinuses is essential and must before inserting antibiotic impregnated nail.

According to pharmacokinetic studies, antibiotic delivery from antibiotic impregnated bone cement is 200 times higher concentration than systemic administration without any systemic side effects. Local high antibiotic concentration not only prevents bacterial growth but also prevents bio-film formation by bacteria. The study was conducted to evaluate the efficacy of antibiotic-impregnated PMMA cemented v-nails in managing infected IL Nailing tibia without any bony defects.

Methods: They were 10 males and 02 females, with a mean age of 38.5 years. Mean follow-up was of 24 months (range 15-30 months). Mean duration for union was patients were followed up at 4, 8, 12 weeks and 3, 6, 9 and 12 months interval. Functional results were evaluated with regards to control of infection, bony union, deformity, limb length discrepancy and complications (intra and post-operative).

Results: Radiological and functional outcome assessed according to complications treated accordingly.

Conclusions: IL nail tibia with infection can be treated effectively if control of infection by debridement and mechanical stability (by v-nail), prevention of bacterial growth by antibiotic impregnated V-nail. Control of infection after debridement is addressed by antibiotic impregnated v-nail is the main crux of treatment. After control of infection cemented V-nail is exchanged with standard IL-nail. Bone grafting is optional, if after exchange nailing there is no sign of union by 8 weeks. Insertion of antibiotic impregnated cemented V-nails combined with adequate debridement; lavage and systemic antibiotic administration significantly contribute to infection control after intramedullary nailing.

Keywords: Antibiotic cement impregnated intramedullary nail, Infected tibia ILN, Non-union tibia, MRSA 


\section{INTRODUCTION}

Tibia is a most common subcutaneous long bone which is highly vulnerable for injury. ${ }^{1}$ As antero-medial surface i.e. shin of tibia is subcutaneous, fractures are most of the times compound fractures. High velocity road traffic accident is associated with significant contamination in compound fractures. ${ }^{2}$ So also tibia is commonest bone which goes into nonunion. Due to these two reasons, infected nonunion of tibia is much common in clinical practice which calls for challenging complex management protocols. Mechanical stability is of primary importance for control of infection. Infection will not be under control unless and until mechanical stability is given. Infected tibia ILN is a complex orthopedic problem. Various treatment modalities are used to control infection and give mechanical stability till infection is eradicated. Later on, definitive fixation and bone grafting is a standard protocol in most of the modalities. Mechanical stability in presence of infection can be given by conventional external fixator OR antibiotic impregnated v-nail OR Ilizarov external fixator.

Conventional external fixator has very high percentage pin-tract infection and pin loosening. Ilizarov technique is not widely practiced routine orthopaedic surgeons are not still expert in doing Ilizarov. Ilizarov fixation gives very good results in infected gap non unions where along with bony union, bone lengthening is desired. ${ }^{3}$

Ilizarov calls for special training and long learning curve, so also non-affordability for implants by poor patients and unavailability of instruments at all set ups. Pin tract infection is more common with tensioned wires in patients who do outdoor jobs in rural areas. Ilizarov technique has best literature record, but not widely practiced due to the reasons mentioned above in all centres.

Paley first introduced antibiotic impregnated nail with satisfactory results. Pharmaco-kinetic study shows, Antibiotic impregnated cement nail gives 200 times more local antibiotic concentration without any systemic side effects, gives mechanical stability, and fills dead spacewhich prevents growth of bacteria and bio-film formation after radical debridement.

In the study antibiotic impregnated cemented $\mathrm{V}$ nail for temporary fixation after radical debridement and reaming was preferred. After 2-3 months when there is no infection, fixation shifted over to final modality of fixation of interlock nailing. After 6-8 weeks of interlock nailing, if necessary, the autogenous cancellous bone grafting was done if there are no signs of radiological union.

Antibiotic impregnated cement beads at wound site after debridement was useful to prevent local bacterial growth.

\section{METHODS}

12 consecutive patients with intramedullary infections of the tibial shaft were studied. They were 10 males and 02 females, with a mean age of 38.5 years.

\section{Inclusion criteria}

All patients with intramedullary infection of tibia fracture treated with IL nail and bone loss of less than $1.0 \mathrm{~cm}$ at fracture site with no union were included.

05 patients had closed fractures initially and 07 patients had an open fracture.

\section{Exclusion criteria}

Patients with more than $1.0 \mathrm{~cm}$ bone loss were excluded from the study.

Functional results were evaluated with regards to control of infection, bony union, deformity, limb length discrepancy and complications (intra and post-operative).

Management is based upon,

- Radical thorough debridement locally at fracture site and thorough reaming and lavage of medullary canal.

- Mechanical stability by Intra-medullary nail/V-nail.

- Antibiotic impregnated cement coating over nail or cement beads at wound site.

\section{Radical thorough debridement}

Removal of sequestrum, infected granulation tissue, infected nail removal and reaming of medullary canal to remove infection from endosteum with serial reaming.

\section{Mechanical stability by intramedullary nail}

All the 12 patients had IM nail in-situ. 10 patients had a discharging sinus at the time of presentation, which averaged 7.1 months, with a range from 3 to 12 months. Out of 08 patients with IM nil in-situ at presentation, 02 patients had pain and inability to bear weight, with X-ray evidence showing no consolidation at fracture site (Figure 1, Figure 6).

04 had radiological evidence of osteolysis on X-rays around nail, locking bolts and periosteam and little/no evidence of union.

\section{Treatment consisted of the following steps}

Patients underwent a pre-operative evaluation which includes: Complete blood count, ESR, C-reactive protein, 
culture and sensitivity. Standard radiographs of the part in two views were also obtained.

\section{Operative technique}

- The infected IM nail was removed first. And a thorough debridement of the fracture site was done.

- Debridement and over-reaming of medullary canal.

- Preparation of antibiotic impregnated indigenous cement nail.

- Insertion of cement impregnated V-nail.

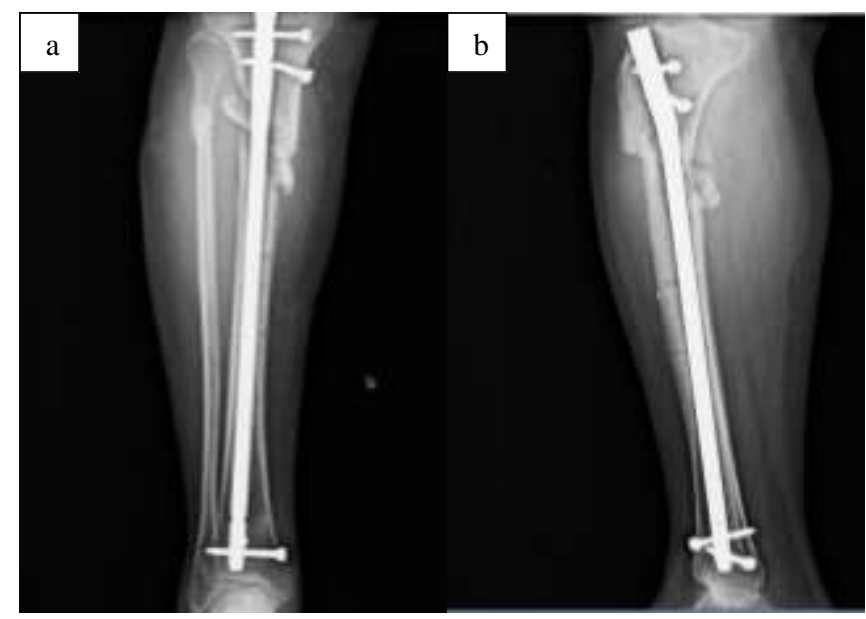

Figure 1: Pre-operative; a) AP view, b) lateral nail insitu.

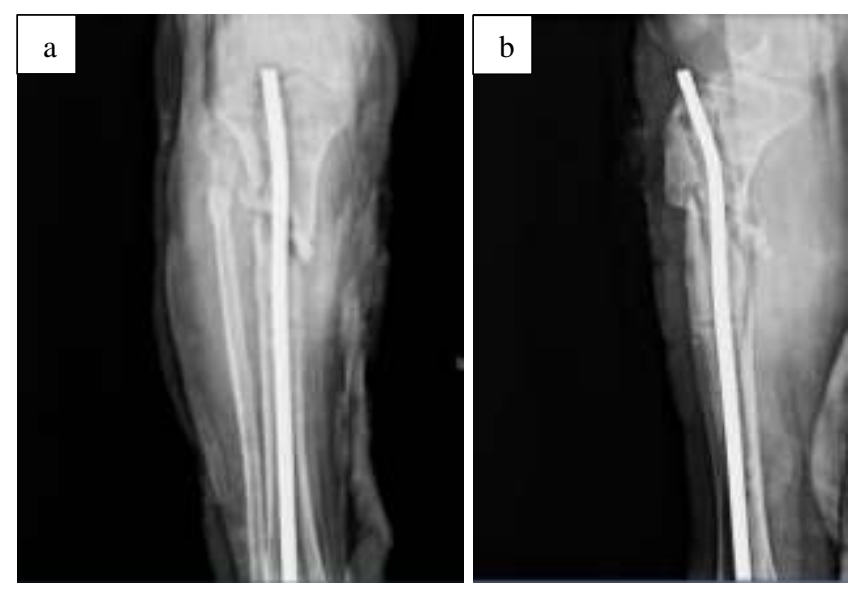

Figure 2: Post-operative; a) AP view, b) lateral v-nail in-situ.

\section{Debridement}

It consists of excision of sinus tracts, scarred and infected soft tissue was performed. This included removal of any sequestrum. All the nonviable and infected tissues, including the skin, soft tissue and bone are excised and infected avascular bony ends were resected, till there was punctuate bleeding from bone ends (paprika sign). Specimens of the bone, soft tissues and any purulent material were sent for culture and antibiotic sensitivity.
The canal was reamed using serial non-flexible reamers. Over-sized reaming was done to remove endosteal infected material, micro-sequestrum and granulation tissue from canal. The reaming material and granulation tissue were sent for repeat cultures to confirm bacteria.

Thorough lavage performed. It includes thorough wash with $\mathrm{H}_{2} \mathrm{O}_{2}$ and povidone iodine twice and normal saline irrigation in the both ends of fracture to wash out necrotic tissue and blood clots.
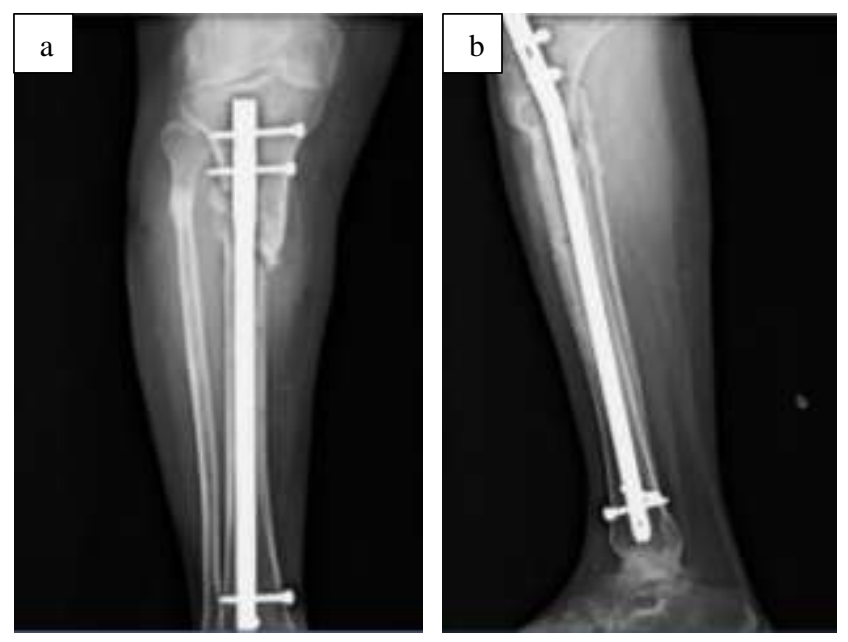

Figure 3: Follow up union at fracture site; a) AP view, b) lateral view.

Preparation of antibiotic impregnated indigenous cement nail

- Teflon tube method-Preferred

- Manual Gauze Method

\section{Teflon tube method}

Preparation of intramedullary canal is an important step. Adequate/oversized reaming is done so as to accommodate a larger diameter nail which ensures more stability. Preferably we ream with $10 \mathrm{~mm}$ size reamer (if feasible sometimes $11 \mathrm{~mm}$.) The limb was prepared and re-draped again before antibiotic cement impregnated nail is prepared. The surgical team changed their gloves and gowns to maintain asepsis before preparing cemented nail.

The nail is prepared on a separate sterile table. Nail length is measured with a guide wire. Antibiotic Impregnated $\mathrm{V}$ nail was prepared with 40 grams of PMMA cement. The antibiotic used was one to which the cultured organism was sensitive to as well as being heat stable. Vancomycin $2 \mathrm{gm}$ and gentamycin $80 \mathrm{mg}$ or cefuroxime $1.5 \mathrm{gm}$ are heat stable and are the preferred antibiotics.

Manual mixing of cement was performed with antibiotics. According to culture and sensitivity report, 
any of 02 antibiotics were chosen. When the cement is in dough state, it was injected into sterilized TEFLON tube of inner diameter of $9 \mathrm{~mm}$ or $10 \mathrm{~mm}$ according to the last reamer passed. $\mathrm{V}$-nail commonly used size is $6-7 \mathrm{~mm}$ of appropriate length.

The v-nail was then inserted in the teflon tube filled with cement. Bone cement is allowed to set for 8-9 minutes. When the cement sets, i.e. it hardens, the teflon tube was cut longitudinally with scalpel blade. The finished antibiotic cement nail was ready for use (Figure 2, 7).

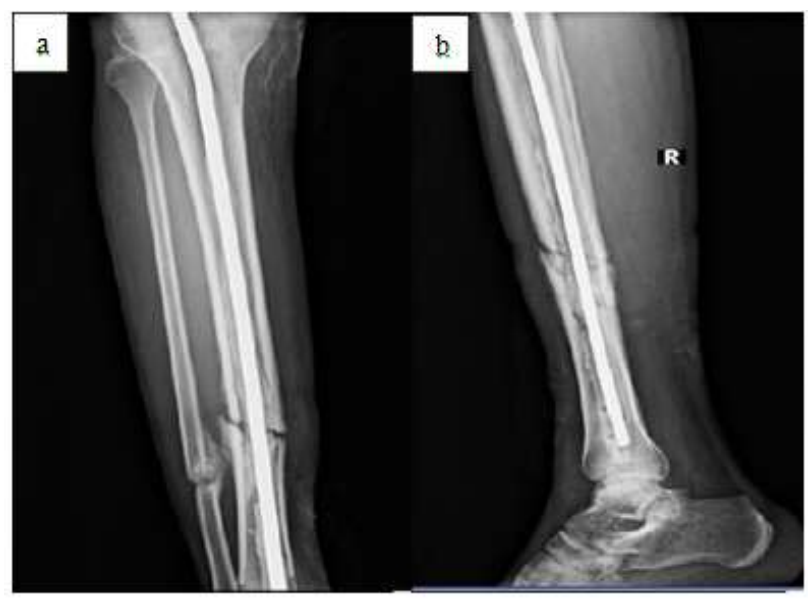

Figure 4: Operated with v-nail in-situ; a) AP view, b) lateral view.

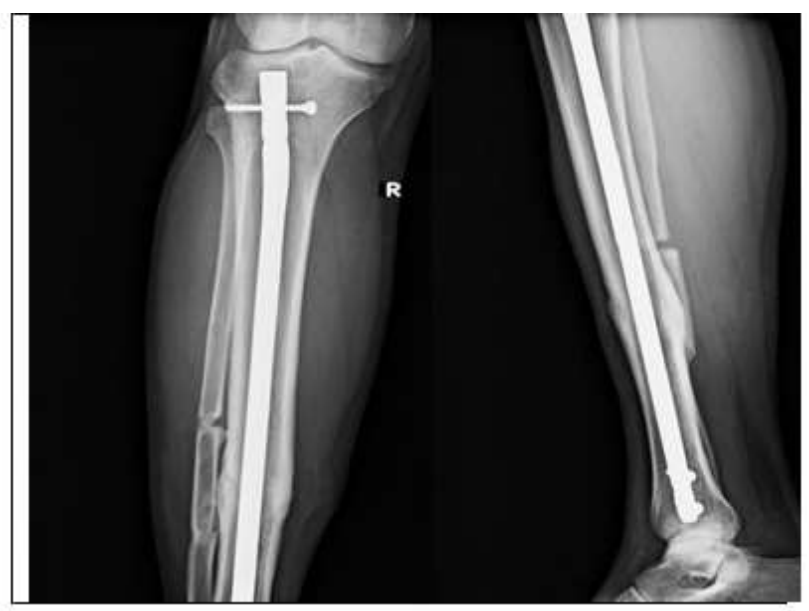

Figure 5: Final union at 14 months.

Teflon tube gives smooth and uniform nail coating of antibiotic impregnated PMMA and thus avoids impaction of nail in medullary canal at the time of removal.

\section{Manual gauze method}

Another method of preparation of cemented v-nail is manual application of cement over circumference of nail. Bone cement was prepared and when it was in dough state, applied manually over the whole surface of v-nail, leaving extraction slot free. Cement is rolled manually to achieve smooth cover encircling the circumference of nail. The prepared cemented nail with cement still in doughy state is passed through gauze meter to check outer diameter. If diameter is more than desired $(1 \mathrm{~mm}$ less than the last reamer size i.e. generally $9-10 \mathrm{~mm}$ ) outer cement is removed manually.

All this has to be completed in precise time i.e. 7-8 minutes by experienced surgeon because, cement sets after 8-9 minutes and becomes stony hard.

An Antibiotic impregnated cemented nail which will impart stability to the bone, as well as release high local concentration of antibiotic into the surrounding tissue was then inserted. ${ }^{4}$

According to Nade's Principle, antibiotic is effective when pus is drained and infected granulation tissue is removed. So also, antibiotics prevent further pus formation as antibiotic cement impregnated nail delivers higher concentration locally without any local/systemic side effects. Pharmaco-kinetic study shows cement impregnated nail or beads delivers 200 times more concentration locally. ${ }^{5}$ Wound is inspected at intervals of 48-72 hour. A repeat debridement was done whenever required. Intravenous broad spectrum antibiotics are given based on culture and sensitivity reports for 2 weeks follower by oral antibiotics for 4 weeks depending on the organism isolated in culture and sensitivity of the aspirated reamed material.

A complete blood count (CBC), erythrocyte sedimentation rate (ESR) and C-reactive protein (CRP) levels were performed at interval of 02 weeks, and 6 weeks.

Clinical and radiological assessment and lab studies were used to assess the progress of control of infection.

Evidence of infection control was gauged mainly clinically and radiologically.

\section{Criteria used were}

- No discharging sinus and dry wound edges.

- Decreased local pain.

- No warmth and tenderness.

- No radiographic evidence of/presence of the osteolytic area.

\section{Definitive fracture fixation}

After confirmation of infection control by clinicoradiological and haematological investigation indicating infection CBC, ESR, CRP levels, the study proceed for definitive fracture stabilization after 8-10 weeks of primary cemented nail insertion. 
The interlocking nail with both static and dynamic locking was carried out as per need of fracture geometry (Figure 3).

In 03 cases, after 08 weeks due to poor healing of bone and gap at the fracture ends on radiological assessment dynamization of long bone fragment was done and union achieved.

Autogenous cancellous bone grafting was done in 05 cases to achieve union when there were no signs of fracture healing after 6-8 weeks of definitive fixation.

Gradual or later on full weight bearing allowed which was protected initially by PTB Cast for 4-6 wks.

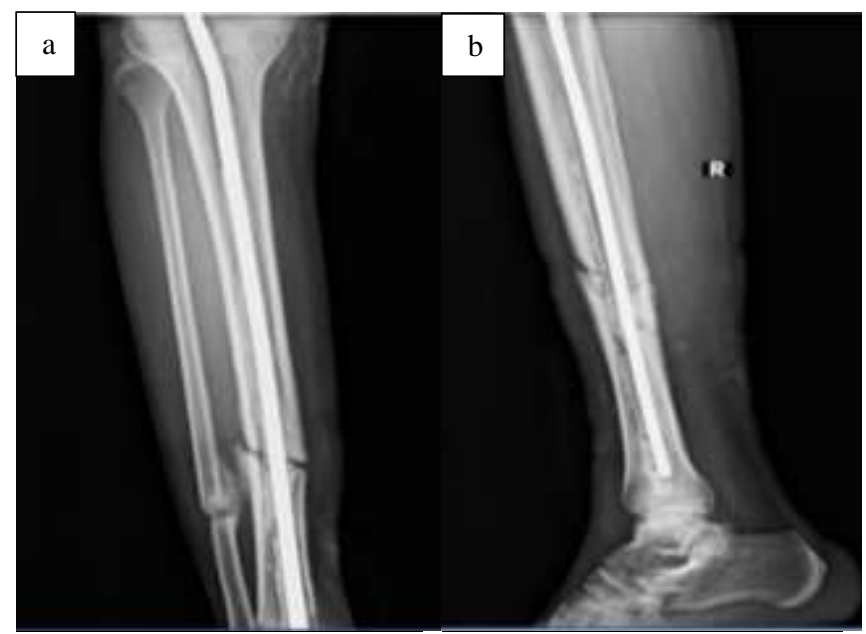

Figure 6: Operated with v-nail in-situ; a) AP view, b) lateral view.

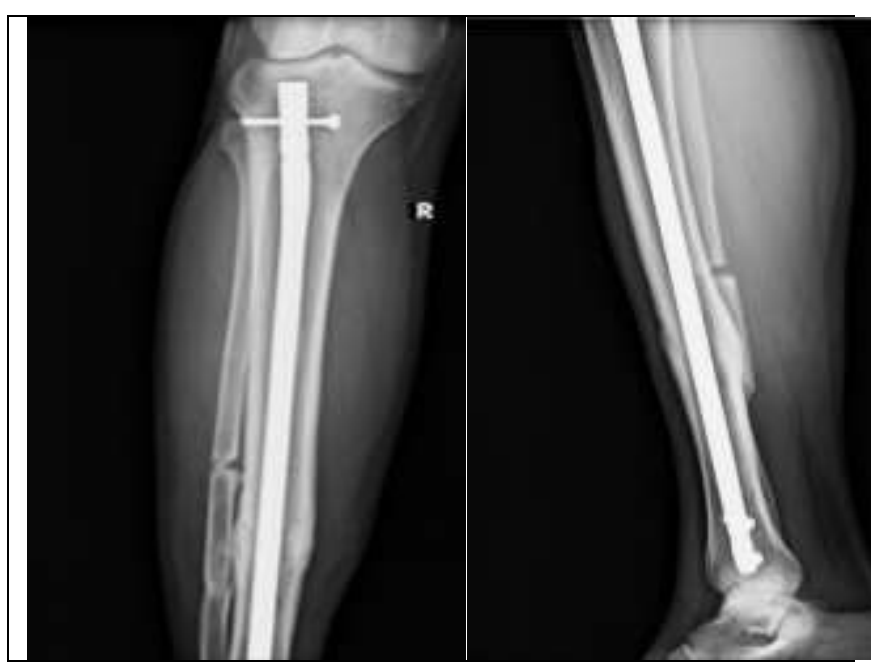

Figure 7: Final union at 14 month.

Mobilization of the patient as per tolerance with active physiotherapy for regaining ankle and knee mobility was advised till the range of movement was satisfactory.

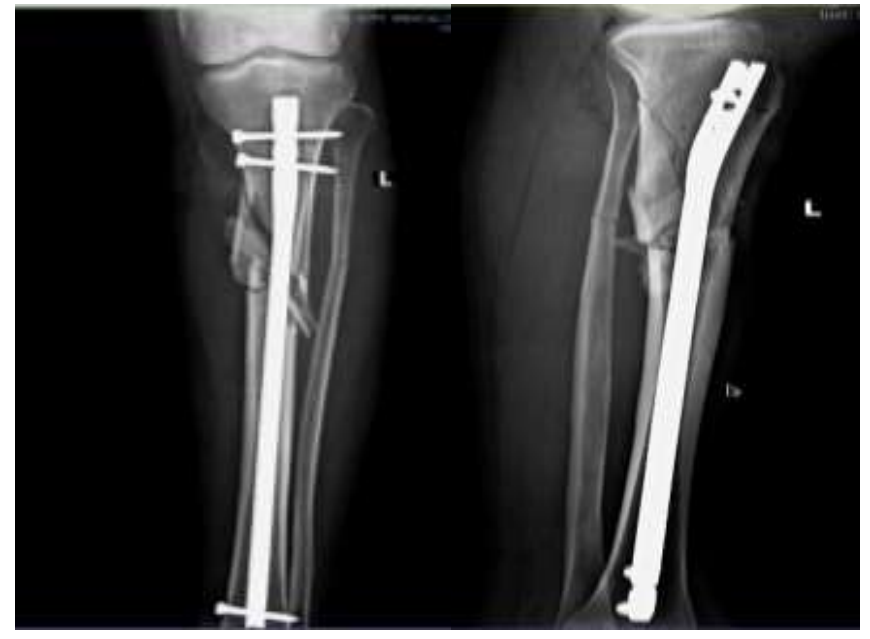

Figure 8: IL nail with infection at 3 months postoperative.

\section{RESULTS}

Intramedullary reaming material sent for culture and antibiotics sensitivity showed that, positive culture of all 10 cases, 02 were culture negative cases.

Per-operatively 07 patients were culture positive for methicillin-resistant Staphylococcus aureus (MRSA) 02 samples were positive for Pseudomonas aeruginosa and 02 cases were positive for citrobacter group of species and 01 case for E. coli.

The mean duration of indigenous cement nail was 8 week ( 6 to 10 weeks). All the patients except one achieved infection control as indicated by laboratory parameters and clinical evaluation. In patients who had no control of infection, the procedure was repeated and after that he was treated with definitive fixation.

Intramedullary cultures were obtained on removal of $\mathrm{V}$ nail and definitive fixation with interlock nailing and the results showed no bacterial growth in all patients.

All the patients were followed up for the average period of 24 months (range 15-30 months). 01 patient was lost in follow up and at the end of 15 months he presented with union with cemented v-nail in situ.

The average time for union was 16 weeks (range, 12- 18 weeks).

- Complications

- Impaction of cement nail.

- Debonding of cement.

- Impaction of cement nail

At the time of nail removal, impaction was common complication. Cemented nail was difficult to remove after 6-8 weeks as there is significant fibrosis around cement. Other possible reasons for impaction are non-uniformity 
of cement mantle. In this study patient presented late i.e. after 12-14 weeks of cemented nail, nail was stucked and impacted in proximal fragment. We used osteotome to debond the cement from nail and removed cement manually. Afterwards nail removal was easy as the stucked cement to the medullar y canal surface was removed.

\section{Debonding of cement}

In one patient there was debonding of cement mantle in distal fragment. Nail removed, fracture site opened and limb elevated at 90 hip flexion with knee full extension. Gentle thumping over heel aided the cement mantle to deliver at proximal end of distal fragment which was removed manually from fracture site.

\section{DISCUSSION}

- The literature about bone infection and osteomyelitis treatment emphasizes upon the importance of extensive surgical debridement in eliminating this infections.6,7 However, surgical debridement and systemic antibiotic treatment without local antibiotic augmentation is associated with a higher rate of recurrence and subsequent operations. 6

- This study demonstrate that using an antibiotic coated cemented nail is simple, cost effective, controls intramedullary infection and promotes fracture union and early mobilization of the patient. In this series, the treatment was monitored through radiographs and clinical wound observations. All patients had complete wound healing. No radiographic evidence/presence of the osteolysis area were noted.

- In present study, maximum of 08 to 10 weeks with antibiotic impregnated v-nail in-situ for control of infection. If the infection was not controlled after 6 weeks the nail was removed and another antibiotic impregnated nail with changed antibiotics as per culture and sensitivity inserted. The antibiotic used was one which was both heat stable and to which the organism was sensitive. These included the cephalosporin, amino glycosides and Vancomycin.

- Observation during the study

- The cemented nail, provides mechanical stability and in addition provides high local concentration of the antibiotic and thus controls bone infection which is pre-requisite for bony union to achieve.

- Advantages of antibiotic cement impregnated nailing
- High local concentration of antibiotic, 200 times greater than systemic drug administration without any systemic side effects. Antibiotics level above the minimal inhibitory concentration of sensitive organisms. Also, we can modify antibiotic as per the culture and sensitivity report.

- Antibiotic concentration at source of infection is not dependent on the pharmacokinetics of the antibiotic. Systemic toxicity of antibiotics is not observed.

- Wider surface area of elution allows for high antibiotic concentration along entire length of the bone. This is essential as necrotic and avascular segments are permeable to systemic par-enteral antibiotic therapy.

- There is a high local concentration of antibiotic even in the presence of extensive scarring and compromised vascularity.

- Does not require use of cumbersome Conventional External fixator OR Ilizarov.

- Patient compliance is found good with this method as IN Ex fix with Ilizarov the apparatus is bulky and prone for pin tract infection (PTI). For this regular follow up is not as cumbersome as for Ex fix.

- Post-operative morbidity is less due to early passive mobilization of limb and it essentially helps in final rehabilitation.

- Long stay in hospital is avoided. Hence the chances for hospital acquired infections are low.

- Patients with elevated renal parameters can be treated with effective concentration of antibiotic without side effects.

- Overall patient compliance in our study was good and acceptance was better when compared with compliance to external fixators in literature.

\section{CONCLUSION}

- IL nail tibia with infection can be treated effectively if control of infection by debridement and mechanical stability (by $\mathrm{V}$ nail), prevention of bacterial growth by antibiotic impregnated $\mathrm{V}$ nail.

- Control of infection after debridement is addressed by antibiotic impregnated $\mathrm{V}$ nail is the main crux of treatment. After control of infection, cemented vnail is exchanged with standard IL-Nail. Bone grafting is optional, if after exchange nailing there is no sign of union by 8 weeks. 
- Insertion of antibiotic impregnated cemented v-nails combined with adequate debridement, lavage and systemic antibiotic administration significantly contribute to infection control after intramedullary nailing.

- Advantages of conventional indigenously prepared cement nail

- It is a safe, patient friendly, versatile procedure that can be adapted easily. The method utilises existing easily available instrumentation and materials to manage a complex problem in a highly cost effective way.

- Antibiotic cement impregnated nailing provides effective infection control and good stability to promote union and has good patient compliance.

- Thus, antibiotic cement impregnated nailing is a simple, economical and very effective procedure when compared to the traditional methods in management of infected IM Nails in fracture tibia.

\section{ACKNOWLEDGEMENTS}

The authors are very grateful to all patients who consented for this study without which the efforts for this study were futile. The study is sincere attempt to treat the patients with minimal invasive methodology and desire to get excellent functional outcome in tertiary care hospital, following strict orthopaedic principles. The authors also acknowledge all para-clinical staff, concerned human efforts towards patient care in this endeavour.
Funding: No funding sources

Conflict of interest: None declared

Ethical approval: Not required

\section{REFERENCES}

1. Boulton C, Robert V, Toole O. Tibia and fibula shaft fractures. In Rockwood and Greens Fracture in adults. $8^{\text {th }}$ edition. Wolters Kluwer Health; 2015:2415.

2. Brown CM, Mcbirnie J. The epidemiology of tibia fractures. J Bone Joint Surg Br. 1995;77(3):417-21.

3. Gregory D. Osteomyelitis In; Terry SC, James HB. Campbell Operative Orthopedics. $12^{\text {th }}$ edition. International edition. Elsevier;2012:739.

4. Dror P, John EH. Intramedullary infections treated with antibiotic cement rods: preliminary results in nine cases. J Orthopaedic Trauma. 2002;16(10):7239.

5. Canale ST, Beaty JH. Campbell Operative Orthopedics. $12^{\text {th }}$ edition. International edition. Elseviers;2012:706.

6. Simpson AH, Deakin M, Latham JM. The effect of the extent of surgical resection on infection-free survival. J Bone Joint Surg Br. 2001;83:403-7.

7. Eckardt JJ, Wirganowicz PZ, Mar T. An aggressive surgical approach to the management of chronic osteomyelitis. Clin Orthop. 1994;298:229-39.

8. Cheng YF, Ming SH, Wei MC. Cheng FC. Successful management of infected intramedullary nailing with reaming, lavage, and insertion of antibiotic-impregnated cement rods. J Exp Clin Med. 2011;3(3):137-41.

Cite this article as: Gawali SR, Barve V, Kukale SB, Niravane PV. Successful management of infected tibia IL nail by debridement, lavage, and antibiotic impregnated v-nail. Int J Res Orthop 2016;2:120-6. 